Western University

Scholarship@Western

2016

\title{
A conjugate fluid-porous approach for simulating airflow in realistic geometric representations of the human respiratory system
}

Christopher DeGroot Dr

Western University

Anthony G. Straatman

uwo, agstraat@uwo.ca

Follow this and additional works at: https://ir.lib.uwo.ca/mechanicalpub

Part of the Materials Science and Engineering Commons, and the Mechanical Engineering Commons

\section{Citation of this paper:}

DeGroot, Christopher Dr and Straatman, Anthony G., "A conjugate fluid-porous approach for simulating airflow in realistic geometric representations of the human respiratory system" (2016). Mechanical and Materials Engineering Publications. 16.

https://ir.lib.uwo.ca/mechanicalpub/16 


\section{A Conjugate Fluid-Porous Approach for Simulating Airflow in Realistic Geometric Representations of the Human Respiratory System}

\author{
Christopher T. DeGroot ${ }^{1}$ \\ Mem. ASME \\ Department of Mechanical and Materials Engineering, \\ Western University, \\ London, ON N6A 5B9, Canada \\ e-mail: cdegroo5@uwo.ca
}

\section{Anthony G. Straatman}

Mem. ASME

Department of Mechanical and Materials Engineering,

Western University,

London, ON N6A 5B9, Canada

e-mail: astraatman@eng.uwo.ca

Simulation of flow in the human lung is of great practical interest as a means to study the detailed flow patterns within the airways for many physiological applications. While computational simulation techniques are quite mature, lung simulations are particularly complicated due to the vast separation of length scales between upper airways and alveoli. Many past studies have presented numerical results for truncated airway trees, however, there are significant difficulties in connecting such results with respiratory airway models. This article presents a new modeling paradigm for flow in the full lung, based on a conjugate fluid-porous formulation where the upper airway is considered as a fluid region with the remainder of the lung being considered as a coupled porous region. Results are presented for a realistic lung geometry obtained from computed tomography (CT) images, which show the method's potential as being more efficient and practical than attempting to directly simulate flow in the full lung. [DOI: $10.1115 / 1.4032113$ ]

\section{Introduction}

The ability to numerically simulate flow in the human lung is of great interest to the medical community because of the potential advancements in respiratory drug delivery, particle deposition, etc., that can be attained with detailed knowledge of the flow patterns within the lung [1]. It is not, however, a simple task to simulate flow in the whole lung because of the many orders of magnitude separating the length scales of the upper airways and the alveoli. The air-filled portion of the lung can be divided into two main regions: (i) the conducting region, which consists of a network of bifurcating airways that transport air to the respiratory region, and (ii) the respiratory region, where gas exchange occurs by passive diffusion through the thin walls of the alveolar sacs which surround the respiratory airways [2]. At the trachea, the first airway generation in the conducting region, the diameter is of the order of centimeters; at the alveolar sacs, the terminal generation in the respiratory region, the diameter is of the order of a fraction of a millimeter [2]. In addition to the large separation of length scales, there are approximately $300 \times 10^{6}$ alveolar sacs in the human lung [2], which cannot possibly be considered individually when simulating processes in the lung using the computational technology of today or even the foreseeable future.

\footnotetext{
${ }^{1}$ Corresponding author
}

Manuscript received January 28, 2015; final manuscript received November 27. 2015; published online January 29, 2016. Assoc. Editor: Naomi Chesler.
Computational fluid dynamics (CFD) simulations have been conducted in both the upper airways [1,3-19] and the alveolated ducts within the respiratory region [20-31], however, there is great difficulty in connecting the results at the two levels in order to simulate processes in the whole lung. As computational power has increased over the years, the total number of airway segments considered in upper airway simulations has increased from seven [3] to over 1400 [11] (although the computational mesh was not sufficiently refined in this case to have grid-independent results). That being said, the total number of airway segments in a full 23generation airway tree would be more than $1.6 \times 10^{7}$ and would require hundreds of billions of mesh elements to adequately discretize the domain for simulation $[15,18]$. Since simulations of this size appear to be out of reach for quite some time, other innovative approaches need to be developed to capture the fundamental information of a full lung simulation model with less computational effort.

Several innovative methods for simulating more branches of the airway tree with less effort have been proposed including those that simulate small subsections of the airway tree and use the outlet condition of one subunit as the input to the next subunit $[7,10]$, the use of partially resolved airway trees $[11,15]$, and the coupling of three-dimensional CFD models for the upper airways with one-dimensional resistance models for the lower airways $[1,14]$. In addition to saving computational time, the use of approximate models for smaller airways is quite pragmatic given that the geometric models at such small scales are approximate as well. As a result, it may be argued that from a practical standpoint it is a better use of resources to solve a simplified model in the small airways rather than solving the flow in an approximate geometry with high accuracy.

It has been proposed by Owen and Lewis [32], in their theoretical work on high-frequency ventilation, that the lung parenchyma can be modeled as a porous continuum. Using homogenization and volume-averaging, they developed a model to describe the flow and tissue deformation for small uniform samples of lung tissue. While the theoretical development of their model was wellfounded, it depended on several effective properties of the porous lung parenchyma that were only roughly estimated and only select one-dimensional results were obtained for the flow and tissue deformations. Lande and Mitzner [33] used the equations of Owen and Lewis [32] to develop a model for lung impedance based on parameters describing the properties of the lung.

In light of the preceding discussion, it is the goal of this work to propose a new method for simulating flow in the full lung by treating it as a conjugate fluid-porous domain. We hypothesize that the large upper airways can be treated as a fluid region and the remaining airways and alveoli can be treated as a connected, coupled porous region where the flow is driven by the moving boundary of the lung. The focus of this work is on the development and implementation of a robust mathematical model of a conjugate lung model based on a realistic representative lung geometry, as well as a demonstration of the potential applications of the model. In future studies, it is envisioned that the proposed model could be used for more comprehensive parameter studies, alongside experimental measurements.

In this article, the governing equations and the associated numerical methods will first be outlined, followed by the setup of the problem, including the method for obtaining the lung geometry from CT images, details of creating a meshed lung model, parameter estimation, and boundary conditions. Finally, results are presented using the coupled fluid-porous model of the lung solved using the proposed numerical method. Ultimately, it is shown that the proposed modeling paradigm has potential as an efficient tool for simulating processes in the full human lung.

\section{Methods}

2.1 Governing Equations and Numerical Methods. It is proposed that the lung may be considered as a conjugate 
fluid-porous domain, where the fluid region is a truncated airway tree and the porous region is the remainder of the lung. In the fluid region, the flow is governed by the continuity and Navier-Stokes equations for an incompressible fluid, given as

$$
\nabla \cdot \mathbf{u}=0
$$

and

$$
\rho_{f}\left[\frac{\partial \mathbf{u}}{\partial t}+\nabla \cdot(\mathbf{u u})\right]=-\nabla p+\mu_{f} \nabla^{2} \mathbf{u}
$$

respectively. In the porous region, flow is governed by the volume-averaged counterparts of Eqs. (1) and (2), given as

$$
\nabla \cdot\langle\mathbf{u}\rangle=-\frac{\partial \varepsilon}{\partial t}
$$

and

$$
\rho_{f}\left[\frac{\partial\langle\mathbf{u}\rangle}{\partial t}+\nabla \cdot\left(\frac{1}{\varepsilon}\langle\mathbf{u}\rangle\langle\mathbf{u}\rangle\right)\right]=-\varepsilon \nabla\langle p\rangle^{f}+\mu_{f} \nabla^{2}\langle\mathbf{u}\rangle-\frac{\varepsilon \mu_{f}}{K}\langle\mathbf{u}\rangle
$$

respectively, where a detailed derivation of Eqs. (3) and (4) can be found in Ref. [34]. For the purposes of this work, it is assumed that the orientation of the ducts is random such that the flow has no preferred direction within the parenchyma, such that the permeability can be taken as a scalar. The Reynolds number is taken to be sufficiently low that non-Darcy effects are negligible, which is certainly reasonable in the respiratory airways. To further simplify the governing equations, spatial variation of porosity is not included, although its addition is relatively straightforward to implement numerically after expansion of the convection term by the product rule. The temporal variation in porosity is retained in the governing equations, although porosity is taken as being constant in time for the case considered herein.

At the interface between the fluid and porous regions, the equations are coupled by appropriate conditions which ensure a balance of fluxes, as well as a balance of viscous and pressure forces. The basic numerical approach used in this work follows that presented previously by the authors in Ref. [35], which has been shown to be an effective method of coupling fluid and porous regions where general unstructured grids are required to discretize the domain of interest.

Since breathing is driven by the motion of the boundary of the lung, the computational mesh must be considered to be in motion. As a result, some extensions to the original numerical method presented in Ref. [35] must be made in order to reformulate the method in an arbitrary Lagrangian-Eulerian (ALE) framework, which is briefly described below. Since the fluid is incompressible, the mesh motion does not impact the continuity equations [36], so only the momentum equations will be discussed in light of ALE framework. Rewriting the momentum equations in integral form for an arbitrary control volume in space, $\Omega$, which is bounded by the moving control surface $\partial \Omega$, results in $[36,37]$

$$
\frac{\partial}{\partial t} \int_{\Omega} \rho_{f} \mathbf{u} d V+\int_{\partial \Omega} \rho_{f} \mathbf{u}\left(\mathbf{u}-\mathbf{u}_{s}\right) \cdot \mathbf{n} d S=-\int_{\partial \Omega} p \mathbf{n} d S+\int_{\partial \Omega} \mu_{f} \nabla \mathbf{u} \cdot \mathbf{n} d S
$$

$$
\begin{aligned}
& \text { and } \\
& \begin{aligned}
\frac{\partial}{\partial t} \int_{\Omega} \rho_{f}\langle\mathbf{u}\rangle d V & +\int_{\partial \Omega} \frac{\rho_{f}}{\varepsilon}\langle\mathbf{u}\rangle\left(\langle\mathbf{u}\rangle-\mathbf{u}_{s}\right) \cdot \mathbf{n} d S=-\int_{\partial \Omega} \varepsilon\langle p\rangle^{f} \mathbf{n} d S \\
& +\int_{\partial \Omega} \mu_{f} \nabla\langle\mathbf{u}\rangle \cdot \mathbf{n} d S-\int_{\Omega} \frac{\varepsilon \mu_{f}}{K}\langle\mathbf{u}\rangle d V
\end{aligned}
\end{aligned}
$$

where the unit-normal vector to the surface $\partial \boldsymbol{\Omega}$ is denoted $\mathbf{n}$, and $\mathbf{u}_{s}$ is the velocity of the control surface, which is used to account for mesh motion.
It is important when calculating flows on moving meshes that the surface velocity, $\mathbf{u}_{s}$, is selected in such a way that volume is conserved in order to avoid artificial mass sources in the domain. This concept is expressed through the "geometric conservation law" [36-40], which is taken into account in the discretization of the governing equations for this work. Discretization of the governing equations is carried out using a spatially second-order finite-volume method, as described in Ref. [35], with the appropriate modifications to the transient and convection terms to account for the changing cell volume and control surface velocity [36,37].

In addition to discretization of the governing equations, a procedure for updating the locations of the mesh nodes, based on prescribed boundary motions, is required. In this work, the motion of the mesh nodes is determined by numerically solving Laplace's equation with a variable diffusion coefficient and Dirichlet conditions on all domain boundaries [41], which is reasonably robust for large deformations, is relatively straightforward to implement, and is not overly expensive to compute. The Laplace equation is solved using a cell-centered finite-volume method, similar to that used to solve all other transport equations in this work, where the mesh stiffness coefficient is taken to be inversely proportional to the cell volume such that larger cells absorb more of the motion and smaller cells move more like rigid bodies.

2.2 CT Image Segmentation. Low-dose whole lung CT images obtained from a single breath hold with a $1.25 \mathrm{~mm}$ slice thickness were obtained from the ELCAP Public Lung Image Database. ${ }^{2}$ From these images, a three-dimensional representation of the airway tree, up to a certain bifurcation, and a representation of the remaining lung volume were obtained. The truncated airway tree was extracted using the segmentation software ITK-SNAP [42], however, only a limited number of bifurcations were captured to prevent the segmented volume from leaking into regions of the lung outside of the airways. The resulting segmentation was exported as a stereolithography (STL) file and was smoothed and decimated, to reduce the total number of facets, using MESHLAB. ${ }^{3}$

The remaining lung volume was segmented by first using the automatic threshold segmentation feature in OSIRIX [43], however, due to limitations in the segmentation algorithm, it was not possible to exclude the airway tree from the segmented volume. Thus, the airways were manually removed from the segmented volume and any geometric problems were manually repaired. The segmentation obtained in OSIRIX was then exported as a new image series and segmented using ITK-SNAP [42], which produced significantly smoother surfaces than OSIRIX. Again, MESHLAB was used to smooth and decimate the exported faceted surface.

2.3 Geometric Model and Meshing. After segmentation, the STL surfaces generated for the airway tree and the remaining lung volume were imported into ANSYS ${ }^{\circledR}$ ICEM CFD, Release 13.0, to be combined, further cleaned up, and meshed for CFD simulations. The airway and lung volume surfaces were first intersected and the portion of the lung surface penetrated by the airway tree was removed. Additionally, the ends of the airway tree branches and trachea were modified such that they formed flat surfaces. The resulting surface model is shown in Fig. 1. From this figure, it is obvious that the $\mathrm{CT}$ images did not include details of the mouth and larynx. If one were to conduct simulations of purely inspiratory flow in a truncated airway tree, this configuration would not necessarily pose a problem since a standard inlet boundary condition could be applied where the trachea is truncated. However, in this case, a full breathing cycle with both inspiratory and expiratory flow is to be considered and the flow is to be driven by the motion of the boundary. Therefore, the opening where the trachea is truncated must allow both inflow and outflow, possibly simultaneously when the bulk flow is changing direction. As a result, the

\footnotetext{
http://www.via.cornell.edu/databases/lungdb.html

${ }^{3} \mathrm{http}: / /$ meshlab.sourceforge.net/
} 


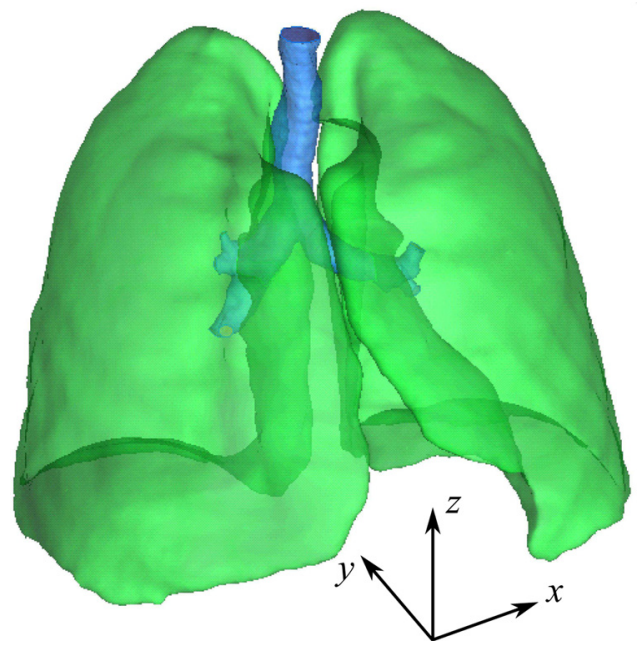

Fig. 1 An illustration of the combined airway tree (inner structure) and the lung surfaces (remaining structure)

trachea was extended somewhat to allow the flow to develop more fully before exiting the domain, and a hemispherical cap was added to mimic expansion to an open environment. Treatment of this boundary will be discussed further in Sec. 2.6.

The resulting geometric model was meshed in ANSYs ${ }^{\circledR}$ ICEM CFD, Release 13.0, using tetrahedral volumes that were refined near fluid-porous interfaces as well as at the airway walls and the lung boundary. The meshed model is shown in Figs. 2(a) and 2(b) which show the main lung volume and the trachea extension, respectively.

2.4 Grid Motion. To provide boundary conditions for the Laplace equation governing the mesh motion, an estimate of the breathing rate and the nature of the lung motion are required. For the test cases considered, a breathing rate of 12 breaths per minute was chosen, which is within the normal range of breathing rates [44]. The motion of the lung, which drives breathing, is a result of the motion of the diaphragm, a thin sheet of muscle located along the bottom surface of the lung [44]. Although there is also motion of the ribcage, it has been shown that the magnitude of the motion of the diaphragm is somewhat larger [45]. Thus, for simplicity, it is assumed for the purposes of this work that all motions are driven by the diaphragm and that the magnitude of its motion is $1.5 \mathrm{~cm}$, consistent with normal breathing [44]. It is found that this specification of the lung motion results in a tidal volume of approximately $400 \mathrm{~mL}$, which is slightly smaller than the typical tidal volume of $500 \mathrm{~mL}$ given by West [44], thus represents light breathing. It should be noted at this point that the lung motion is simply a boundary condition to the mesh motion algorithm and is thus easily modified if one wishes to explore more elaborate specifications of its motion to reflect a specific breathing pattern.

Although it has been stated that the mesh motion at the diaphragm should be $1.5 \mathrm{~cm}$, the mesh motion must be specified on all boundaries in order to solve for the motion of the interior nodes. In this case, the motion of all boundaries nodes is taken to be in the $z$-direction, as shown in Fig. 1. The magnitude of the displacement should be $1.5 \mathrm{~cm}$ at the base of the lung, whereas at the top of the lung it should be zero. Thus, an appropriate variation between these two values must be specified. To avoid motion of the truncated airways, the motion is blended between a value of zero and $1.5 \mathrm{~cm}$ in the region below the truncated airways.

2.5 Parameter Estimation. According to Weibel's idealized "model A" lung geometry, more than three quarters of the volume of air in the lung resides in generations 20-23, which are fully alveolated ducts or alveolar sacs [2]. Further, almost $90 \%$ of the

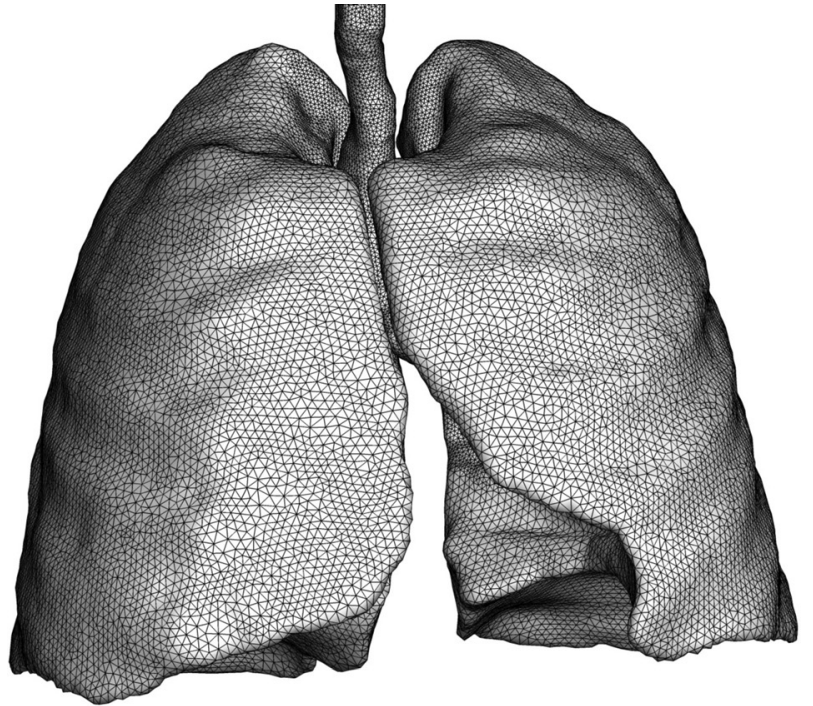

(a)

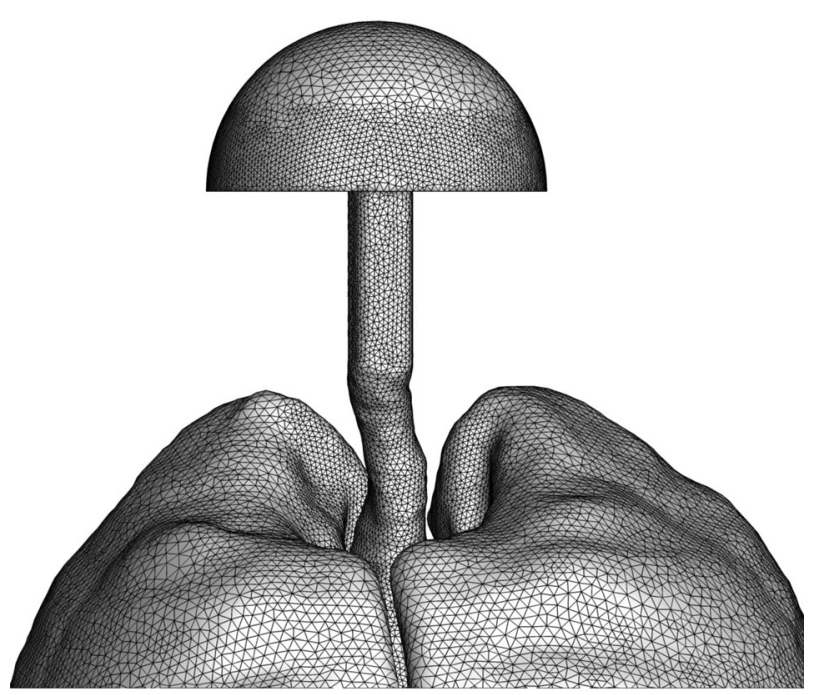

(b)

Fig. 2 Plots of the computational meshes for the lung geometry showing (a) the main lung mesh and $(b)$ the trachea extension

air resides in generations 17-23 which have at least some degree of alveolation [2]. Thus, a logical approximation for the permeability of the lung parenchyma is the permeability of an alveolated duct, which has been determined numerically in Ref. [46]. Taking a volume-weighted average of the airway diameters in generations 17-23 given by Weibel [2] yields an average diameter of $0.43 \mathrm{~mm}$. Using this average diameter and the result for the dimensionless permeability given in Ref. [46] yields an estimate for the average permeability of the lung parenchyma of $K$ / $\varepsilon=1.75 \times 10^{-9} \mathrm{~m}^{2}$, where the porosity remains to be determined.

According to the measurements of Gehr et al. [47], the lung is composed of $86.5 \%$ air, $5.7 \%$ capillary blood, and $7.8 \%$ tissue. The same study also found that this composition does not change significantly in space, justifying the previous assumption of zero spatial porosity gradient. Results of Kampschulte et al. [48] indicate significant variations in air volume fractions between subjects, with measurements ranging from approximately 81-93\%. Therefore, a rough estimate of $\varepsilon=0.9$ is used for the purposes of this work and is taken as constant in time for simplicity, noting that extension to a time-varying porosity is included in the formulation. 
Based on the value $\varepsilon=0.9$, the permeability is $K=1.58 \times 10^{-9} \mathrm{~m}^{2}$. Note that this permeability estimate is for a representative airway diameter, which will change with expansion and contraction of the lung. For the purposes of this work, it will be assumed that the hydraulic diameter of the duct varies sinusoidally with time, according to

$$
D_{H}=D_{H, 0}\left[1+\Lambda_{D_{H}} \cos (\omega t)\right]
$$

where $D_{H, 0}$ is the hydraulic diameter at mean inflation, and $\Lambda_{D H}$ is the dimensionless amplitude of the change in hydraulic diameter. This leads to the permeability as a function of time, given as

$$
K=K_{0}\left[1+\Lambda_{D_{H}} \cos (\omega t)\right]^{2}
$$

where $K_{0}$ is the permeability at mean inflation, i.e., $K_{0}=1.58 \times 10^{-9} \mathrm{~m}^{2}$.

According to the studies by Sznitman et al. [24] and Harding and Robinson [28], the volume of an alveolated duct changes by approximately $15 \%$ from minimum to maximum inflation, indicating that the length scale of the duct changes by about $5 \%$. Accordingly, the amplitude of the change in duct diameter is taken to be $\Lambda_{D_{H}}=0.025$, such that $D_{H}$ varies between $0.975 D_{H}$ and $1.025 D_{H}$.

It should be noted that the parameter estimates described in this section are meant simply to provide the model with data that are of approximately the right order of magnitude in order to assess the reasonableness of the final results. For more detailed studies, or those specific to a particular patient, more accurate values for these parameters should be obtained.

2.6 Boundary Conditions. The walls of the airway tree are considered as fixed no-slip, impenetrable walls, such that all velocity components may be set to zero and the pressure is extrapolated from the interior of the domain to the boundary. Note that the walls of the extended trachea and the flat lower surface of the hemispherical cap are treated in the same way as fixed walls. The lung boundary is considered to be a moving no-slip, impenetrable wall and the velocity components are selected such that there is no mass flux through any of the control surfaces on the moving walls. On the moving walls, the pressure is also extrapolated from the interior of the domain.

At the open boundary, i.e., the curved portion of the hemispherical cap, the derivatives of all velocity components normal to the boundary are set to zero. When the fluid is exiting the domain, a constant static pressure is specified, whereas when the fluid enters

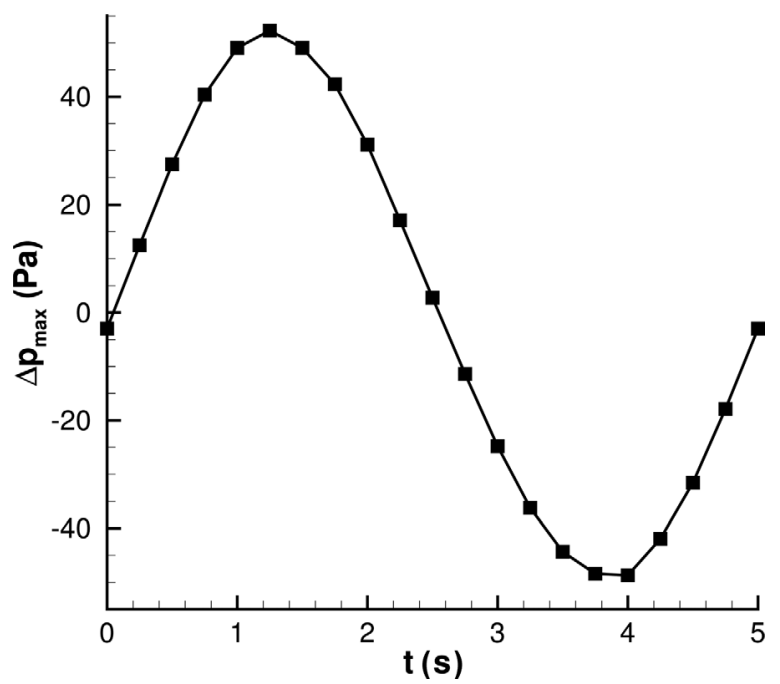

Fig. 3 A plot of the maximum pressure difference within the domain as a function of time, where $t=0$ represents the beginning of the breath cycle at maximum inflation the domain, a constant dynamic pressure is specified, as recommended by Mathur and Murthy [49] for open boundaries.

\section{Results and Discussion}

3.1 Scope. The model for air flow in the human lung described in Sec. 2 was run for five full breath cycles to ensure that any initial transient behavior was dissipated and results were extracted from the final full breath cycle that was computed. For the purposes of the results to be presented, the time $t=0$ corresponds to the beginning of the final breath cycle. The computational mesh shown previously in Fig. 2 contained 911,156 tetrahedral control volumes. In this section, results will be presented for the maximum pressure difference across the domain, representing the pressure drop from the trachea to the most distal alveolus, as well as pressure contours on the surface of the lung and velocity vectors in a plane intersecting the bifurcation from the trachea to the main bronchi. Although detailed experimental

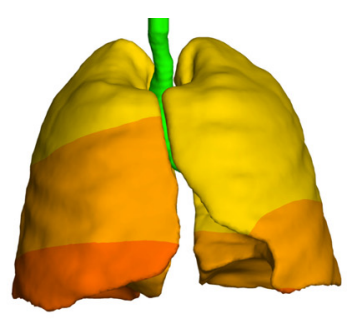

(a)

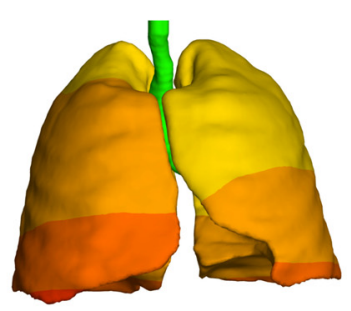

(c)

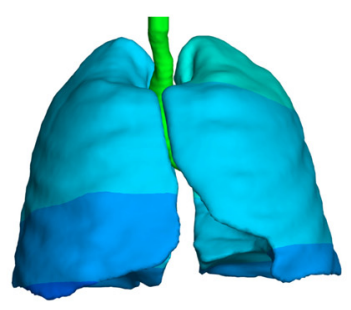

(e)

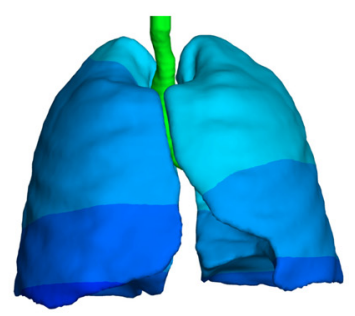

(g)

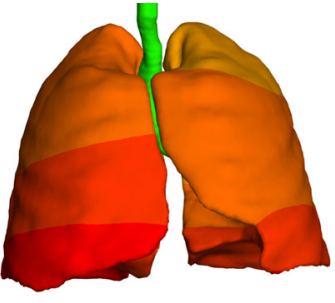

(b)

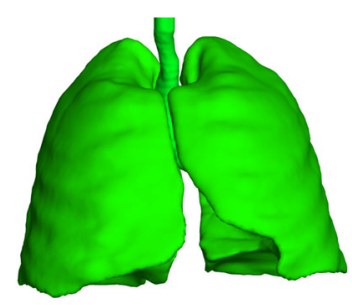

(d)

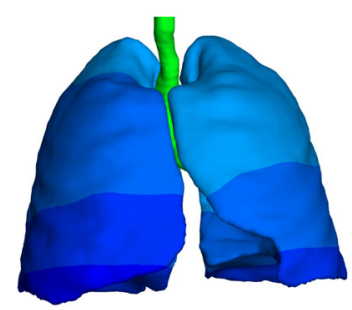

(f)

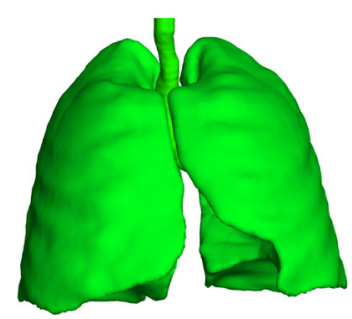

(h)
$-45-40-35-30-25-20-15-10 \quad-5 \quad 0 \quad 5 \quad 10 \quad 15 \quad 20 \quad 25 \quad 30 \quad 35 \quad 40 \quad 45$

Fig. 4 Contour plots of the pressure in Pascals for the times: (a) 0.75 , (b) 1.25, (c) 1.75, (d) 2.5, (e) 3.25, (f) 3.75, (g) 4.25, and

(h) $5.0 \mathrm{~s}$ from the beginning of the breath cycle 


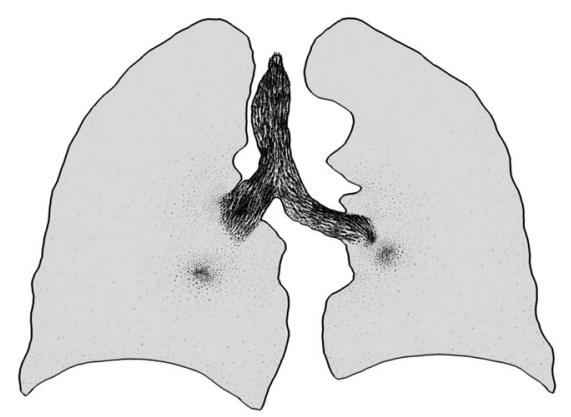

(a)

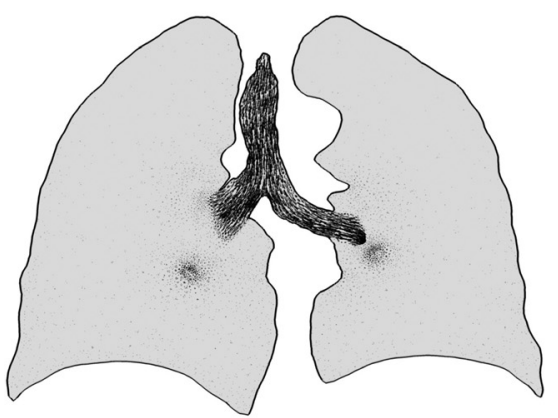

(c)

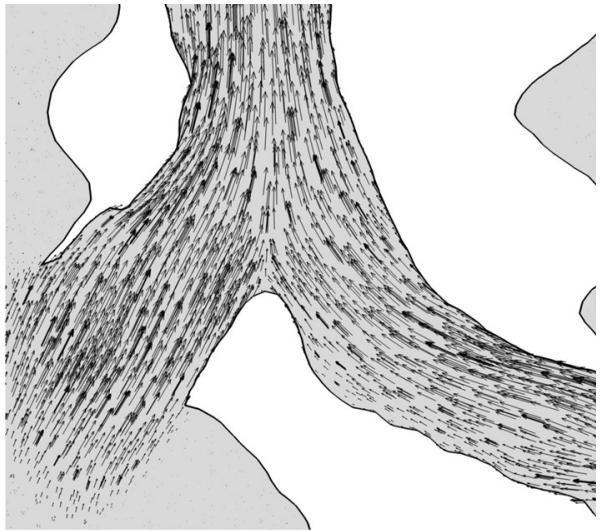

(b)

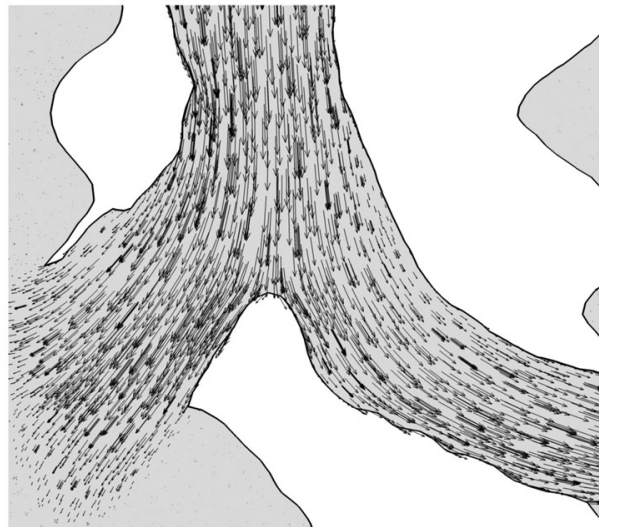

(d)

Fig. 5 Contour plots of velocity vectors in the $x y$-plane, at times: (a) and (b) 1.25 and (c) and (d) $3.75 \mathrm{~s}$ from the beginning of the breath cycle

data for comparison are not yet available, connections will be made with typical values for pressure drop in the lung.

3.2 Alveolar Pressure. Figure 3 shows the maximum pressure difference across the domain, representing the pressure drop from the inlet surface to the most distal alveolus, where the pressure difference is maximum. Note the maximum magnitude of the alveolar pressure shown in this figure is just over $50 \mathrm{~Pa}$, which is the correct order of magnitude for a healthy lung which has a pressure drop on the order of $1 \mathrm{~cm} \mathrm{H}_{2} \mathrm{O}(98 \mathrm{~Pa})$ for normal breathing [44]. Since the breathing simulated here is light breathing with only $80 \%$ of the tidal volume as compared to normal breathing, this is indeed the correct magnitude for the pressure drop. Certainly, this increases confidence in the validity of this approach and the method of estimating the permeability, although experimental verification should be pursued in the future.

3.3 Pressure Field. Contour plots of the pressure on the surface of the lung model are given in Fig. 4 for various times within the breath cycle. This figure illustrates the higher pressures exhibited during expiration near the diaphragm where the lung motion is largest in Figs. 4(a)-4(c). In its fully contracted state, shown in Fig. 4(d), and in its fully expanded state, shown in Fig. 4(h), the pressure is nearly zero everywhere since there is no net flow in or out of the lung. In Figs. 4(e)-4(g), it is shown that the pressure is lower near the base of the lung as the air is drawn into the lung. In all cases, it can be seen that the pressure within the trachea is similar.

3.4 Velocity Field. Velocity vectors at two time points are shown in Fig. 5 in an $x z$-plane that intersects a portion of the trachea and the region where the trachea bifurcates into the main bronchi. Figures $5(a)$ and $5(b)$ show the vectors for the time $t=1.25 \mathrm{~s}$ from the beginning of the breath cycle (peak expiratory flow rate), while Figs. $5(c)$ and $5(d)$ show the vectors for the time $t=3.75 \mathrm{~s}$ from the beginning of the breath cycle (peak inspiratory flow rate). These figures show the velocity magnitude dropping significantly after the main bronchi bifurcate, such that the velocity vectors are hardly visible. Within the porous region, the velocity is clearly very small as expected for the respiratory region. Note the bursts of velocity shown in Figs. 5(a) and 5(c) which occur where the slice intersects a smaller airway included in the fluid region.

3.5 Comparison with Direct CFD Approach. With the results presented, it is important to put into perspective the advantages of the proposed approach to simulating processes in the lung. In comparison to direct simulations in truncated airway trees, the proposed approach has the clear advantage of being able to consider both an upper airway tree and the remainder of the lung through the porous media model. Additionally, the computational cost of adding the porous region is significantly less than adding enough airway branches to the direct CFD model to capture the full lung or any significant part of the respiratory region. Note that the current model employed 911,156 control volumes and that a fully resolved airway tree would require an estimated $7.2 \times 10^{8}$ control volumes $[15,18]$.

As another comparison, consider the recent work of Gemci et al. [11] which simulated 1433 individual airway branches across 17 generation of the human lung. While this is far from the more than 100,000 branches actually present in 17 airway generations, the $6.7 \times 10^{6}$ control volumes used to discretize the domain 
(the upper limit possible on the given hardware) was far from sufficient for obtaining grid-independent results. To estimate the number of control volumes actually required, consider the work of Yin et al. [17] who were able to obtain grid-independent results in a realistic 4-5 generation airway geometry using $4.6 \times 10^{6}$ control volumes. Recently, Walters and Luke [15] proposed a novel stochastic coupling method to reduce the number of flow paths that must be simulated directly, although up to $4.3 \times 10^{6}$ control volumes were still required to discretize an eight-generation airway tree model.

Although the proposed method is also somewhat computationally expensive, taking on the order of a few days of computational time (depending on convergence criteria, timestep selection, hardware, etc.) to compute five full breath cycles using 32 processors in parallel, there still appears to be an advantage in terms of reduced computational requirements that can be attained in comparison to the previously mentioned approaches. Even with significant grid refinement and the addition of several more airway generations, it is doubtful that the current model would need to approach the number of control volumes required for reasonably extensive airway tree computations and certainly not the requirements for a full airway tree model.

\section{Conclusions}

In summary, the development of a numerical method for computing flows in conjugate fluid-porous domains with moving boundaries has been presented and applied to the simulation of flow in a complex, physiologically realistic geometry of the human lung. This approach provides a convenient method of simulating processes in the full lung that may be considerably more efficient than simulating flow in large airway trees, since as the airways become smaller and more numerous, the computational costs increase dramatically. The porous media approach is applicable in any case where only the averaged flow and pressure fields are needed in the alveolar region (e.g., studies of gas exchange), but would not be appropriate in cases where the details of the flow in small airways and alveoli are required (e.g., studies of particle deposition where the flow velocities are required to compute particle trajectories). At this stage, the conjugate fluid-porous approach shows promise as an efficient method of simulating air flows in the lung, given that the predicted pressure drop across all airways is reasonable, however, detailed comparisons to experimental measurements should be considered in the future for validation or calibration purposes.

\section{Acknowledgment}

The authors wish to acknowledge the financial support provided by the Natural Sciences and Engineering Research Council (NSERC) and the computing facilities provided by the Shared Hierarchical Academic Research Computing Network (SHARCNET) ${ }^{4}$ and Compute/Calcul Canada.

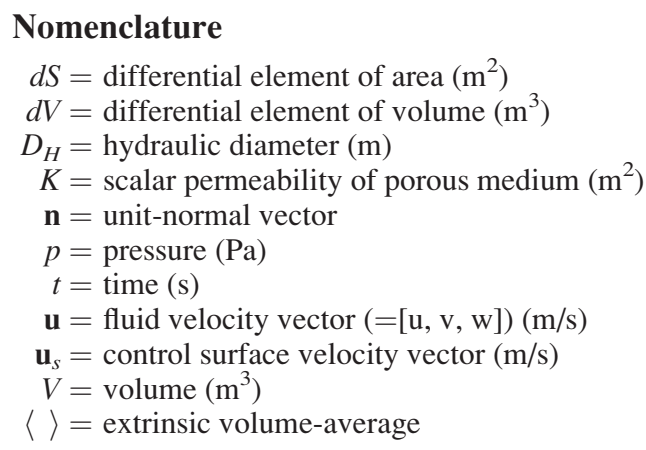

${ }^{4}$ www.sharcnet.ca \langle\rangle$^{f}=$ intrinsic volume-average with respect to the fluid constituent

\section{Greek Symbols}

$\varepsilon=$ porosity $\left(=V_{f} / V\right)$

$\Lambda=$ amplitude

$\mu=$ dynamic viscosity $(\mathrm{kg} / \mathrm{m} \mathrm{s})$

$\rho=$ density $\left(\mathrm{kg} / \mathrm{m}^{3}\right)$

$\omega=$ angular frequency $(\mathrm{rad} / \mathrm{s})$

$\Omega=$ arbitrary control volume in space

$\partial \Omega=$ boundary of the control volume $\Omega$

\section{Subscripts and Superscripts}

$$
\begin{aligned}
& f=\text { fluid } \\
& 0=\text { initial value }
\end{aligned}
$$

\section{References}

[1] Tawhai, M. H., and Lin, C.-L., 2010, "Image-Based Modeling of Lung Structure and Function," J. Magn. Reson. Imaging, 32(6), pp. 1421-1431.

[2] Weibel, E. R., 1963, Morphometry of the Human Lung, Springer-Verlag, New York.

[3] Comer, J. K., Kleinstreuer, C., Hyun, S., and Kim, C. S., 2000, "Aerosol Transport and Deposition in Sequentially Bifurcating Airways," ASME J. Biomech. Eng., 122(2), pp. 152-158.

[4] Zhang, Z., Kleinstreuer, C., and Kim, C. S., 2001, "Flow Structure and Particle Transport in a Triple Bifurcation Airway Model," ASME J. Fluids Eng., 123(2), pp. 320-330.

[5] Zhang, Z., and Kleinstreuer, C., 2002, "Transient Airflow Structures and Particle Transport in a Sequentially Branching Lung Airway Model," Phys. Fluids, 14(2), pp. 862-880.

[6] Zhang, Z., and Kleinstreuer, C., 2003, "Species Heat and Mass Transfer in a Human Upper Airway Model," Int. J. Heat Mass Transfer, 46(25), pp. 4755-4768.

[7] Nowak, N., Kakade, P. P., and Annapragada, A. V., 2003, "Computational Fluid Dynamics Simulation of Airflow and Aerosol Deposition in Human Lungs," Ann. Biomed. Eng., 31(4), pp. 374-390.

[8] van Ertbruggen, C., Hirsch, C., and Paiva, M., 2005, "Anatomically Based Three-Dimensional Model of Airways to Simulate Flow and Particle Transport Using Computational Fluid Dynamics," J. Appl. Physiol., 98(3), pp. 970-980.

[9] Ma, B., and Lutchen, K. R., 2006, "An Anatomically Based Hybrid Computational Model of the Human Lung and Its Application to Low Frequency Oscillatory Mechanics," Ann. Biomed. Eng., 34(11), pp. 1691-1704.

[10] Zhang, Z., Kleinstreuer, C., and Kim, C. S., 2008, "Airflow and Nanoparticle Deposition in a 16-Generation Tracheobronchial Airway Model," Ann. Biomed. Eng., 36(12), pp. 2095-2110.

[11] Gemci, T., Ponyavin, V., Chen, Y., Chen, H., and Collins, R., 2008, "Computational Model of Airflow in Upper 17 Generations of Human Respiratory Tract," J. Biomech., 41(9), pp. 2047-2054.

[12] Luo, H. Y., and Liu, Y., 2008, "Modeling the Bifurcating Flow in a CTScanned Human Lung Airway," J. Biomech., 41(12), pp. 2681-2688.

[13] Nazridoust, K., and Asgharian, B., 2008, "Unsteady-State Airflow and Particle Deposition in a Three-Generation Human Lung Geometry," Inhal. Toxicol., 20(6), pp. 595-610.

[14] Lin, C., Tawhai, M. H., Lennan, G. M., and Hoffman, E. A., 2009, “Multiscale Simulation of Gas Flow in Subject-Specific Models of the Human Lung," IEEE Eng. Med. Biol. Mag., 28(3), pp. 25-33.

[15] Walters, D. K., and Luke, W. H., 2010, "A Method for Three-Dimensional Navier-Stokes Simulations of Large-Scale Regions of the Human Lung," ASME J. Fluids Eng., 132(5), p. 051101.

[16] Comerford, A., Förster, C., and Wall, W. A., 2010, "Structured Tree Impedance Outflow Boundary Conditions for 3D Lung Simulations," ASME J. Biomech. Eng., 132(8), p. 081002.

[17] Yin, Y., Choi, J., Hoffman, E. A., Tawhai, M. H., and Lin, C.-L., 2010, "Simulation of Pulmonary Air Flow With a Subject-Specific Boundary Condition,” J. Biomech., 43(11), pp. 2159-2163.

[18] Walters, D. K., and Luke, W. H., 2011, "Computational Fluid Dynamics Simulations of Particle Deposition in Large-Scale Multigenerational Lung Models,' ASME J. Biomech. Eng., 133(1), p. 011003.

[19] Saksono, P. H., Nithiarasu, P., and Sazonov, I., 2012, "Numerical Prediction of Heat Transfer Patterns in a Subject-Specific Human Upper Airway," ASME J. Heat Transfer, 134(3), p. 031022.

[20] Tsuda, A., Henry, F. S., and Butler, J. P., 1995, "Chaotic Mixing of Alveolated Duct Flow in Rhythmically Expanding Pulmonary Acinus,” J. Appl. Physiol., 79(3), pp. 1055-1063.

[21] Darquenne, C., and Paiva, M., 1996, “Two- and Three-Dimensional Simulations of Aerosol Transport and Deposition in Alveolar Zone of Human Lung," J. Appl. Physiol., 80(4), pp. 1401-1414.

[22] Lee, D. Y., and Lee, J. W., 2003, "Characteristics of Particle Transport in an Expanding or Contracting Alveolated Tube," J. Aerosol Sci., 34(9), pp. 1193-1215. 
[23] Karl, A., Henry, F. S., and Tsuda, A., 2004, "Low Reynolds Number Viscous Flow in an Alveolated Duct," ASME J. Biomech. Eng., 126(4), pp. 420-429.

[24] Sznitman, J., Heimsch, F., Heimsch, T., Rusch, D., and Rösgen, T., 2007, "ThreeDimensional Convective Alveolar Flow Induced by Rhythmic Breathing Motion of the Pulmonary Acinus," ASME J. Biomech. Eng., 129(5), pp. 658-665.

[25] Tsuda, A., Henry, F. S., and Butler, J. P., 2008, "Gas and Aerosol Mixing in the Acinus," Respir. Physiol. Neuro., 163(1), pp. 139-149.

[26] Sznitman, J., Heimsch, T., Wildhaber, J. H., Tsuda, A., and Rösgen, T., 2009, "Respiratory Flow Phenomena and Gravitational Deposition in a ThreeDimensional Space-Filling Model of the Pulmonary Acinar Tree," ASME J. Biomech. Eng., 131(3), p. 031010.

[27] Kumar, H., Tawhai, M. H., Hoffman, E. A., and Lin, C.-L., 2009, "The Effects of Geometry on Airflow in the Acinar Region of the Human Lung," J. Biomech., 42(11), pp. 1635-1642.

[28] Harding, E. M., and Robinson, R. J., 2010, "Flow in a Terminal Alveolar Sac Model With Expanding Walls Using Computational Fluid Dynamics," Inhal. Toxicol., 22(8), pp. 669-678.

[29] Li, Z., and Kleinstreuer, C., 2011, "Airflow Analysis in the Alveolar Region Using the Lattice-Boltzmann Method," Med. Biol. Eng. Comput., 49(4), pp. 441-451.

[30] Ma, B., and Darquenne, C., 2011, "Aerosol Deposition Characteristics in Distal Acinar Airways Under Cyclic Breathing Conditions," J. Appl. Physiol., 110(5), pp. $1271-1282$.

[31] Kumar, H., Tawhai, M. H., Hoffman, E. A., and Lin, C.-L., 2011, "Steady Streaming: A Key Mixing Mechanism in Low-Reynolds-Number Acinar Flows," Phys. Fluids, 23(4), p. 041902.

[32] Owen, M. R., and Lewis, M. A., 2001, "The Mechanics of Lung Tissue Under High-Frequency Ventilation," SIAM J. Appl. Math., 61(5), pp. 1731-1761.

[33] Lande, B., and Mitzner, K. R. W., 2006, "Analysis of Lung Parenchyma as a Parametric Porous Medium,” J. Appl. Physiol., 101(3), pp. 926-933.

[34] DeGroot, C. T., and Straatman, A. G., 2012, "Numerical Results for the Effective Flow and Thermal Properties of Idealized Graphite Foam," ASME J. Heat Transfer, 134(4), p. 042603.

[35] DeGroot, C. T., and Straatman, A. G., 2011, "A Finite-Volume Model for Fluid Flow and Nonequilibrium Heat Transfer in Conjugate Fluid-Porous Domains Using General Unstructured Grids," Numer. Heat Transfer, Part A, 60(4), pp. 252-277.

[36] Demirdzić, I., and Muzaferija, S., 1995, "Numerical Method for Coupled Fluid Flow, Heat Transfer and Stress Analysis Using Unstructured Moving Meshes With Cells of Arbitrary Topology," Comput. Methods Appl. Mech. Eng., 125(1-4), pp. 235-255.
[37] Demirdzić, I., and Perić, M., 1990, "Finite Volume Method for Prediction of Fluid Flow in Arbitrarily Shaped Domains With Moving Boundaries," Int. J. Numer. Methods Fluids, 10(7), pp. 771-790.

[38] Thomas, P. D., and Lombard, C. K., 1979, "Geometric Conservation Law and Its Application to Flow Computations on Moving Grids," AIAA J., 17(10), pp. 1030-1037.

[39] Demirdzić, I., and Perić, M., 1988, "Space Conservation Law in Finite Volume Calculations of Fluid Flow," Int. J. Numer. Methods Fluids, 8(9), pp. 1037-1050.

[40] Venkatakrishnan, V., and Mavriplis, D. J., 1996, "Implicit Method for Computation of Unsteady Flows on Unstructured Grids," J. Comput. Phys., 127(2), pp. 380-397.

[41] Tuković, Z., and Jasak, H., 2012, "A Moving Mesh Finite Volume Interface Tracking Method for Surface Tension Dominated Interfacial Fluid Flow," Comput. Fluids, 55, pp. 70-84.

[42] Yushkevich, P. A., Piven, J., Hazlett, H. C., Smith, R. G., Ho, S., Gee, J. C., and Gerig, G., 2006, "User-Guided 3D Active Contour Segmentation of Anatomical Structures: Significantly Improved Efficiency and Reliability," Neuroimage, 31(3), pp. 1116-1128.

[43] Rosset, A., Spadola, L., and Ratib, O., 2004, "OsiriX: An Open-Source Software for Navigating in Multidimensional DICOM Images," J. Digit. Imaging, 17(3), pp. 205-216.

[44] West, J. B., 2008, Respiratory Physiology: The Essentials, Lippincott Williams \& Wilkins, Baltimore, MD.

[45] Werner, R., Ehrhardt, J., Schmidt, R., and Handels, H., 2008, "Modeling Respiratory Lung Motion-A Biophysical Approach Using Finite Element Methods," Proc. SPIE, 6916, p. 69160N.

[46] DeGroot, C. T., 2012, "Numerical Modelling of Transport in Complex Porous Media: Metal Foams to the Human Lung," Ph.D. thesis, University of Western Ontario, London, ON, Canada.

[47] Gehr, P., Bachofen, M., and Weibel, E. R., 1978, "The Normal Human Lung: Ultrastructure and Morphometric Estimation of Diffusion Capacity," Respir. Physiol., 32(2), pp. 121-140.

[48] Kamschulte, M., Schneider, C. R., Litzbauer, H. D., Tscholl, D., Schneider, C. Zeiner, C., Krombach, G. A., Ritman, E. L., Bohle, R. M., and Langheinrich, A. C., 2013, "Quantitative 3D Micro-CT Imaging of Human Lung Tissue," Fortschr. Röntgenstr., 185(9), pp. 869-876.

[49] Mathur, S. R., and Murthy, J. Y., 1997, "Pressure Boundary Conditions for Incompressible Flow Using Unstructured Meshes," Numer. Heat Transfer B, 32(3), pp. 283-298. 